\title{
KBV-Ziel: gleiches Honorar für alle
}

- In einer außerordentlichen Vertreterversammlung hat die Kassenärztliche Bundesvereinigung (KBV) ein neues Ziel beschlossen: In fünf Jahren soll jeder Arzt in Deutschland für die Lösung des gleichen Patientenproblems die gleiche Vergütung bekommen. Damit reagiert die KBVSpitze auf extrem unterschiedliche Honorarveränderungen je nach Kassenärztlicher Vereinigung nach der letzten Vergütungsreform. Eine Kostenerstattung könnte es ermöglichen, bundesweit das Honorar der Vertragsärzte zu homogenisieren.

Solange es beim Sachleistungssystem bleibt, sind regional unterschiedliche, auf historischen Daten beruhende Leistungsmengen die Ursache für Honorarunterschiede. Folglich muss es eine objektive umfassende Morbiditätsmessung geben. Ansatzweise funktioniert dies bislang nur für Morbiditätsveränderungen, nicht jedoch für die Errechnung eines auch re- gional differenzierten Morbiditätssockels. In diesem Zusammenhang kommt den neuen, ab 2011 von Vertragsärzten anzuwendenden Codierrichtlinien eine strategische Bedeutung zu: Sie sollen die Morbidität objektivieren und die Verhandlungsstrategie der KBV durch Fakten unterfüttern.

Hoffnung auf Abschaffung der Regresse verspricht sich die KBV vom neuen Arzneisparpaket der Koalition.

\section{KBV will Aussteigerquote bei angehenden Ärzten reduzieren}

- Was muss sich in Klinik und Praxis ändern, damit wieder mehr Medizinstudenten in die Patientenversorgung gehen? Das will die Kassenärztliche Bundesvereinigung (KBV) über eine Befragung herausfinden, die an allen Medizinfakultäten gestartet werden soll. Zusammen mit der Universität Trier werde daher an einem entsprechenden Fragebogen gearbeitet, sagte KBV-Vorstand Dr. Andreas Köhler in seiner Rede vor der Vertreterversammlung.

In vielen Gesprächen mit Medizinstudenten sei ihm klar geworden, dass die heutige Generation andere Ansprüche hat als frühere, so Köhler. An den Bewerberzahlen liege es nicht. Auf jeden Studienplatz kämen immer noch vier Bewerber. Köhlers Vermutung: „Die Gründe für den hohen Schwund müssen vorwiegend im Studium selbst liegen und in den Berufsaussichten, die den Studierenden während des Studiums vermittelt werden." Mit den derzeit etwa 82.000 Studenten in der Medizin müsse theoretisch der künftige Bedarf an Ärzten gedeckt werden. Allerdings beendeten nur etwa $82 \%$ der Studierenden das Studium. Von ihnen meldeten sich $12 \%$ nicht bei der Ärztekammer an. 10\% ließen sich zwar registrieren, arbeiteten dann aber bei Pharmaunternehmen, Behörden oder etwa dem Medizinischen Dienst der Krankenkassen.

Um dem drohenden Ärztemangel Herr zu werden, müsse ein ganzes Bündel von Maßnahmen ergriffen werden. Das beginne beim Studienzugang, setze sich fort über die Inhalte des Studiums bis hin zu Praktika in der Praxis niedergelassener Kollegen. Eine Landarztquote, wie sie Bundesgesundheitsminister Philipp Rösler vorgeschlagen hat, findet ausdrücklich die Zustimmung des KBV-Chefs. Hauptproblem sei allerdings die Bedarfsplanung, die komplett überarbeitet werden müsse - und zwar sektorübergreifend: Denn die bisherige Bedarfsplanung fußte auf zu großräumigen Planungsbezirken, einer mangelnden Differenzierung bei der Einteilung der Arztgruppen sowie auf der Nichtberücksichtigung der Versorgungslage in benachbarten Planungsbereichen. Eine neue Bedarfsplanung müsse daher prospektiv ausgerichtet sein und vor allem die Morbiditätsentwicklung in der Region berücksichtigen. $\quad$ vdb 